\title{
Quantum-Dash Mode-Locked Laser as a Source for 56-Gb/s DQPSK Modulation in WDM Multicast Applications
}

Yousra Ben M'Sallem, Quang Trung Le, Laurent Bramerie, Quoc-Thai Nguyen, Eric Borgne, Pascal Besnard, Alexandre Shen, François Lelarge, Sophie LaRochelle, Leslie A. Rusch, and Jean-Claude Simon

\begin{abstract}
We investigate wavelength tunable $56-\mathrm{Gb} / \mathrm{s}$ differential quaternary phase-shift keying (DQPSK) systems using comb generation in a quantum-dash mode-locked laser for wavelength-division-multiple access (WDM)-based broadcast application. We present relative intensity noise and bit-error-rate measurements for each mode. We demonstrate error-free operation over nine WDM channels with 100-GHz spacing.
\end{abstract}

Index Terms-Differential quaternary phase-shift keying (DQPSK) modulation, mode-locked lasers, optical networks, quantum-dash (QD) laser.

\section{INTRODUCTION}

$\mathbf{T}$ HE large scale computer networks needed to support growing web applications require fast and reliable interconnections in order to provide rapid access to information and services to millions of users. As the number of interconnected servers and storage media increases, optical technologies are expected to play a major role due to the high speed, low loss and small foot print of optical fiber communication links. The next generation of optical networks requires efficient, densely packed wavelength division multiple access (WDM) channels to transmit massive amounts of information. An important challenge is the cost and the wavelength versatility of WDM transmitters.

Manuscript received August 12, 2010; revised November 15, 2010; accepted December 03, 2010. Date of publication January 13, 2011; date of current version March 16, 2011. This work was supported by the French National Research Agency projects TELDOT and PERSYST II, by Québec's Regroupement Stratégique Centre for Advanced Systems and Technologies in Communications (SYTACom), and by the Canadian Institute for Photonic Innovations (CIPI).

Y. Ben M'Sallem, S. LaRochelle, and L. A. Rusch are with the Université Laval, Centre for Optics, Photonics and Lasers (COPL), Electrical and Computer Engineering Department, Québec, QC G1V 0A6, Canada (e-mail: yousra. ben-msallem.1@ulaval.ca; sophie.larochelle@gel.ulaval.ca; rusch@gel.ulaval. ca).

Q. T. Le, L. Bramerie, Q.-T. Nguyen, E. Borgne, P. Besnard, and J.-C. Simon are with the Université Européenne de Bretagne (UEB), CNRS-Foton Laboratory (UMR 6082)/Université de Rennes 1, Enssat, BP 80518, 22305 Lannion Cedex, France (e-mail: le@enssat.fr; Laurent.Bramerie@enssat.fr; quocthai.nguyen@enssat.fr; Eric.Borgne@enssat.fr; pascal.besnard@enssat.fr; jean-claude.simon@enssat.fr).

A. Shen is with the Alcatel Thales III-V Laboratory, 91767 Palaiseau cedex, France (e-mail: alexandre.shen@3-5lab.fr).

F. Lelarge is with the Alcatel Thales III-V Laboratory, 91460 Marcoussis, France (e-mail: francois.lelarge@3-5lab.fr).

Color versions of one or more of the figures in this letter are available online at http://ieeexplore.ieee.org.

Digital Object Identifier 10.1109/LPT.2011.2106116
Comb generation in a mode-locked laser (MLL) has been proposed as a cost-effective solution for WDM transmission. The multiple frequencies (modes) of the MLLs offer several advantages compared to sets of single mode distributed feedback (DFB) lasers, including fixed channel spacing (set by the MLL mode spacing), reduced channel crosstalk due to the coherence of the MLL modes, etc. [1].

Different configurations based on mode locked lasers have been demonstrated for optical frequency comb generation. Examples include amplitude or frequency modulated mode-locked lasers, e.g., Er:Yb:glass laser oscillators [2], fiber ring lasers [3] or mode-locked semiconductor lasers [4]. Semiconductor quantum-dot or quantum-dash mode-locked lasers are been heavily investigated owing to their low-noise, high thermal stability, and a broad gain spectrum [5]. Quantum-dash mode-locked lasers (QD-MLL) capable of extremely high bit rates $(100 \mathrm{~Gb} / \mathrm{s})$ [6] are of particular interest. They were first considered for WDM using on-off keying (OOK) [7]. The use of the QD-MLL source as an optical transmitter for colorless WDM passive optical networks (PON) was reported in [8].

Recently, all-optical WDM multicast has attracted much research interest in the field of optical switching and networking, since it can support increasing demands for applications such as video on demand, broadcasting of high definition TV (HDTV), and online video conferencing. In fact, WDM multicasting provides large bandwidth and a high degree of data transparency to the modulation format and the bit rate [9]. In this letter, we propose, for the first time to our knowledge, the use of a quantum-dash mode-locked laser (QD-MLL) as multiwavelength source for WDM multicast application with differential quaternary phase-shift keying (DQPSK) transmission at high bit rate. We experimentally demonstrate the use of nine WDM ITU channels with a channel spacing of $100 \mathrm{GHz}$ for $56 \mathrm{~Gb} / \mathrm{s}$ DQPSK modulation. We show that relative intensity noise (RIN) and phase noise over all modes are compatible with PSK modulation when we modulate the entire QD-MLL spectrum and compare the obtained results with an external cavity laser (ECL) source.

\section{QD-MLL CHARACTERIZATION}

The QD laser structure was grown by gas source molecular beam epitaxy on S-doped (100) InP wafer. The active area consists of 6 InAs-based QD layers. To achieve 100-GHz channel spacing for WDM comb generation, a cavity length of $420 \mu \mathrm{m}$ was used as in [6]. Fig. 1 shows the overall emission spectrum of the QD laser taken at a pump current of $120 \mathrm{~mA}$. Due to 


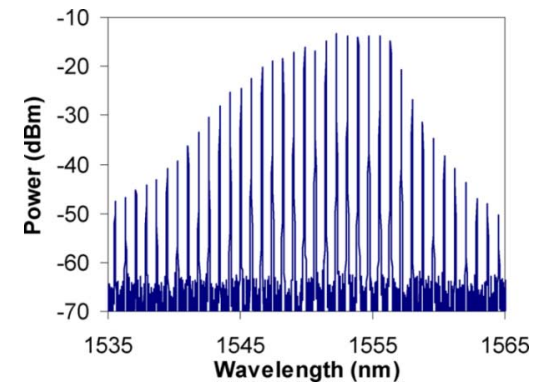

Fig. 1. Optical spectrum of the QD-MLL.

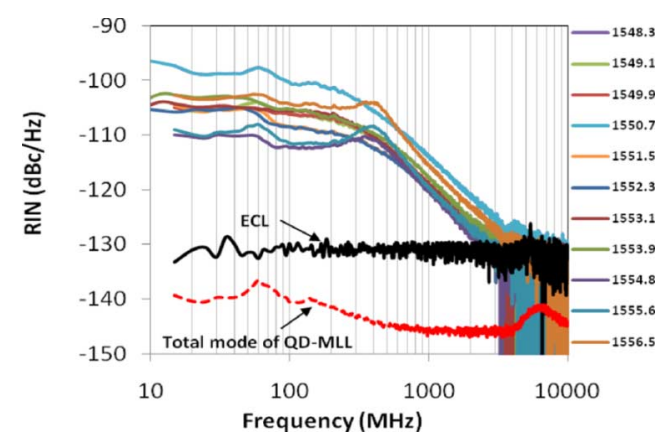

Fig. 2. Relative intensity noise (RIN).

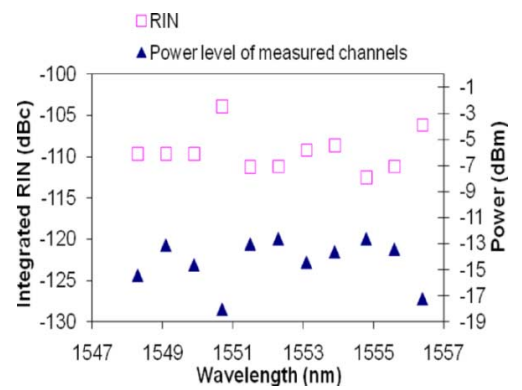

Fig. 3. Power level and RIN of each DQPSK QD-MLL channel.

mode-locking, a flat and wide spectrum centered at $1.55 \mu \mathrm{m}$ is observed. Eleven optical modes, corresponding to 11 ITU channels, are available within the $5-\mathrm{dB}$ optical bandwidth of $8 \mathrm{~nm}$ $(1548.3 \mathrm{~nm}$ to $1556.5 \mathrm{~nm})$.

Fig. 2 shows the RIN measurement results of 1) a commercial ECL, 2) all QD-MLL modes taken together, and 3) each filtered QD-MLL mode (from $1548.3 \mathrm{~nm}$ to $1556.5 \mathrm{~nm}$ ). Due to the mode partition noise, each individual MLL mode has a level of RIN much higher than the combined modes, or that of a singlemode laser [9]. QD lasers have low intensity noise thanks to low amplified spontaneous emission (ASE) [5]. The RIN of the total of all modes is even lower than that of a commercial ECL. As shown in Fig. 2, the modal RIN drops from $-100 \mathrm{dBc} / \mathrm{Hz}$ to $-130 \mathrm{dBc} / \mathrm{Hz}$ in the $0.1-10 \mathrm{GHz}$ range.

Fig. 3 summarizes the RIN integrated in the $0.1-10 \mathrm{GHz}$ range and power level of each QD-MLL mode examined. Not surprisingly, the RIN of the nondominant modes are strong compared to the QD-MLL dominant modes. If the received power is sufficiently high, the bit error rate (BER) is governed by the total RIN. There exists a relationship between RIN and BER for OOK [10]. But in the case of DQPSK, we expect that the penalty for DQPSK may be more nuanced as the linewidth introduces phase noise, while the RIN introduces errors once DQPSK is demodulated into a detected intensity. We will next examine experimentally the impact of RIN on BER performance for DQPSK.

\section{EXPERIMENTAL SETUP}

The experimental setup shown in Fig. 4 consists of a $56 \mathrm{~Gb} / \mathrm{s}$ DQPSK transmitter and receiver. A $100 \mathrm{GHz}$ quantum-dash Fabry-Pérot mode-locked laser source is used at the transmitter. The output of the laser passes through an optical isolator to prevent feedback. The optical spectrum of the QD laser mode is amplified using an EDFA and externally modulated using a DQPSK, LiNbO3 Mach-Zehnder interferometer (MZI). Due to the test equipment availability, we generate two $2^{9}-1$ pseudorandom binary sequences (PRBS) delayed by 256 bits to decorrelate them. At the receiver, an arrayed waveguide grating (AWG) typically demultiplexes the $100-\mathrm{GHz}$ spaced ITU grid channels.

For this experiment, the AWG function is realized by a tunable optical filter with $0.25 \mathrm{~nm} \mathrm{3-dB}$ bandwidth which selects one QD-MLL channel. The spectra for all modulated and filtered QD-MLL lines, immediately before demodulation, are presented in Fig. 5 (spectral resolution is $0.01 \mathrm{~nm}$ ). The spectrally filtered mode is then optically preamplified, filtered and demodulated with delay line interferometers (DLI). The DLIs and balanced detectors convert the optical phase modulation into intensity modulation. The detected signals are demultiplexed by an electronic 1:4 demultiplexer; a $7 \mathrm{~Gb} / \mathrm{s}$ stream is fed to the error detector programmed with the expected differentially demodulated bit patterns. We assumed that the BER is the same on both in-phase (I) and quadrature (Q) branches, and for each tributary.

\section{EXPERIMENTAL RESULTS}

The performance of our system is evaluated for each filtered mode of the QD-MLL. For each wavelength, the DQPSK system is first optimized using the wavelength-tunable ECL source. Next each mode of the QD-MLL is isolated and then BER measurements are performed to compare ECL and QD-MLL performances over the entire $8 \mathrm{~nm}$ range, $(1548.3 \mathrm{~nm}$ to $1556.4 \mathrm{~nm}$ ), covering $11 \mathrm{WDM}$ channels with $100 \mathrm{GHz}$ spacing. Results are shown in Fig. 6. The receiver sensitivity of the system using the ECL varies between $-26 \mathrm{dBm}$ and $-26.5 \mathrm{dBm}$ in this wavelength range. When the QD-MLL is used, error-free performance is obtained over nine channels. Error floors at BER of $4 \times 10^{-7}$ occur with two channels $(1550.7 \mathrm{~nm}$ and $1556.5 \mathrm{~nm})$. This is to be expected given the high level of RIN and low power level of those modes seen in Fig. 3.

Finally, Fig. 7 shows the power penalty (BER of $10^{-9}$ ) of DQPSK signals using each QD-MLL mode. Power penalty from $1.5 \mathrm{~dB}$ to $5 \mathrm{~dB}$ is obtained over nine WDM channels. For the weak modes, the power penalty rapidly increases. Not surprisingly, the RIN fluctuations in nondominant modes are strong compared to that of the dominant modes.

\section{CONCLUSION}

We proposed and experimentally demonstrated for the first time the feasibility of a cost-effective, multiwavelength source based on QD-MLL for high speed, spectrally efficient WDM multicast systems using $56 \mathrm{~Gb} / \mathrm{s}$ DQPSK modulation. We reported error-free operation over nine $C$-band WDM channels with $100 \mathrm{GHz}$ spacing. The multimode QD laser, whose mode 

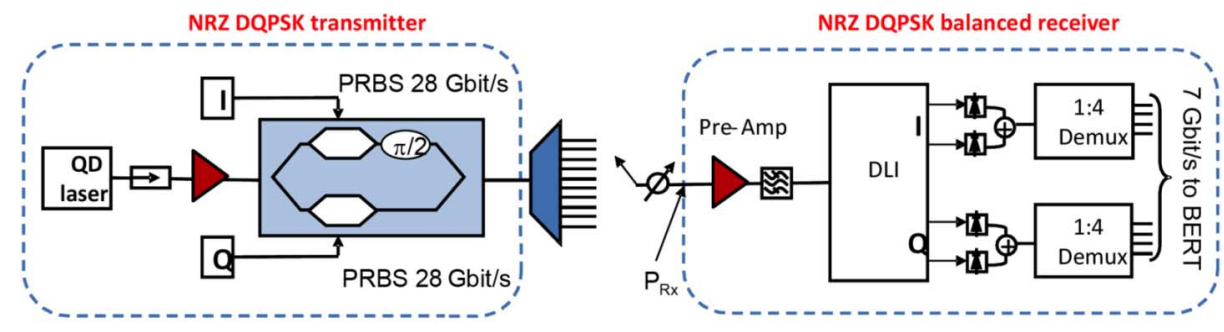

Fig. 4. Experimental setup.

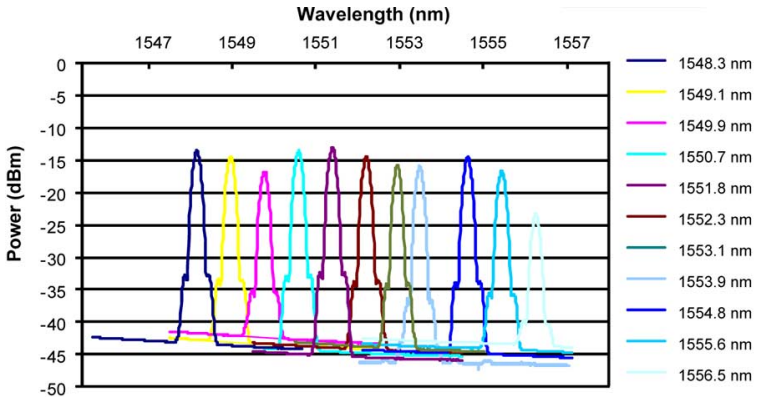

Fig. 5. Optical spectrum of each DQPSK QD-MLL channel.

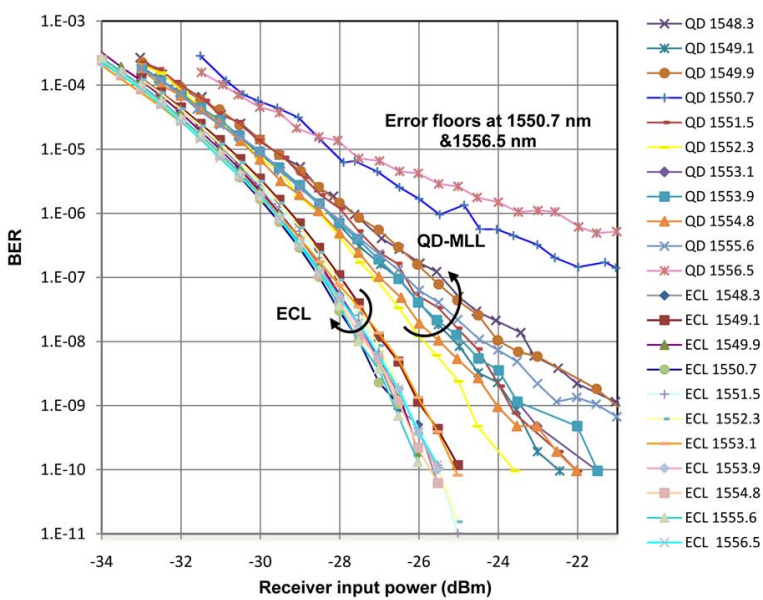

Fig. 6. Bit-error rate versus receiver input power for 11 QD-MLL modes.

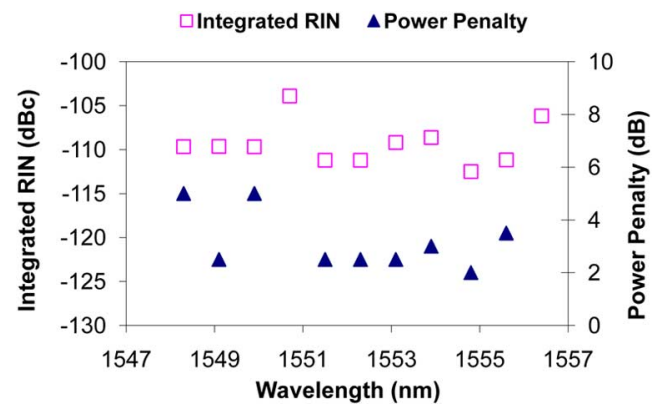

Fig. 7. RIN level and penalty $\left(B E R=10^{-9}\right)$ of DQPSK QD-MLL channels.

spacing is compatible with ITU grids, shows promise for the development of simple and cost-effective high-bandwidth optical solutions.

\section{ACKNOWLEDGMENT}

The experimental test bed was implemented on the Persyst platform at Foton Laboratory. The authors would like to thank P. Chanclou from Orange Laboratory for lending the RIN measurement equipment.

\section{REFERENCES}

[1] T. Healy, F. C. G. Gunning, E. Pincemin, B. Cuenot, and A. D. Ellis, " $1,200 \mathrm{~km} \mathrm{SMF}, 100 \mathrm{~km}$ spans $280 \mathrm{Gbit} / \mathrm{s}$ coherent WDM transmission using hybrid raman/EDFA amplification," in Proc. Eur. Conf. Optical Communications, Berlin, Germany, Sep. 2007, Paper PD 1.9.

[2] S. C. Zeller, G. J. Spühler, L. Krainer, R. Paschotta, U. Keller, and K. P. Hansen, "Frequency comb generation with $50-\mathrm{GHz}$ channel spacing in the telecom C-band," in Proc. Conf. Lasers and Electro-Optics, Munich, 2005.

[3] S. Yamashita and K. Hsu, "Single-frequency, single-polarization operation of tunable miniature erbium:ytterbium fiber Fabry-Perot lasers by use of self-injection locking," Opt. Lett., vol. 23, pp. 1200-1202, 1998.

[4] A. Gubenko, I. Krestinkov, D. Livshtis, S. Mikhrin, A. Kovsh, L. West, C. Bornholdt, N. Grote, and A. Zhukov, "Error-free $10 \mathrm{Gbit} / \mathrm{s}$ transmission using individual Fabry-Perot modes of low-noise quantum-dot laser," Electron. Lett., vol. 43, no. 25, pp. 1430-1431, 2007.

[5] F. Lelarge, B. Dagens, J. Renaudier, R. Brenot, A. Accard, F. van Dijk, D. Make, O. Le Gouezigou, J.-G. Provost, F. Poingt, J. Landreau, O. Drisse, E. Derouin, B. Rousseau, F. Pommereau, and G.-H. Duan, "Recent advances on InAs/InP quantum dash based semiconductor lasers and optical amplifiers operating at $1.55 \mu \mathrm{m}$," IEEE J. Sel. Topics Quantum Electron., vol. 13, no. 1, pp. 111-124, Jan./Feb. 2007.

[6] G. H. Duan, A. Shen, A. Akrout, F. V. Dijk, F. Lelarge, F. Pommereau, O. LeGouezigou, J. G. Provost, H. Gariah, F. Blache, F. Mallecot, K. Merghem, A. Martinez, and A. Ramdane, "High performance InP-based quantum dash semiconductor mode-locked lasers for optical communications," Bell Labs Tech. J., vol. 14, no. 3, pp. 63-84, 2009.

[7] A. Akrout, A. Shen, R. Brenot, F. Van Dijk, O. Lelouezigou, F. Pommerau, F. Lelarge, A. Ramdane, and G.-H. Duan, "Separate error-free transmission of eight channels at $10 \mathrm{~Gb} / \mathrm{s}$ using comb generation in a quantum-dash-based mode-locked laser," IEEE Photon. Technol. Lett., vol. 21, no. 23, pp. 1746-1748, Dec. 1, 2009.

[8] Q. T. Nguyen, L. Bramerie, G. Girault, O. Vaudel, P. Besnard, J. Simon, A. Shen, G. Duan, and C. Kazmierski, " $16 \times 2.5$ Gbit/s downstream transmission in colorless WDM-PON based on injection-locked FabryPerot laser diode using a single quantum dash mode-locked FabryPerot laser as multi-wavelength seeding source," in Proc. Optical Fiber Communication Conf., San Diego, CA, 2009, Paper OThA3.

[9] S. Yoshima, N. Wada, G. Cincotti, T. Miyazaki, and K. Kitayama, "Multicast-capable optical-code label packet switch: Proposal and its experimental demonstration," Opt. Switch. Netw., vol. 5, no. 1, pp. 29-37, Mar. 2008.

[10] Petermann, Laser Diode Modulation and Noise. Norwell, MA: Kluwer, 1991. 